\title{
Determinantes do baixo peso ao nascer em filhos de adolescentes: uma análise hierarquizada
}

\author{
Determinants of low birth weight in the children \\ of adolescent mothers: a hierarchical analysis
}

\author{
Gabriella Pinto Belfort ${ }^{1}$ \\ Marta Maria Antonieta de Souza Santos ${ }^{1}$ \\ Lidiane da Silva Pessoa ${ }^{1}$ \\ Juliana Rebelo Dias ${ }^{1}$ \\ Sonaly Petronilho Heidelmann ${ }^{1}$ \\ Cláudia Saunders ${ }^{1}$
}

${ }^{1}$ Instituto de Nutrição Josué de Castro, Universidade Federal do Rio de Janeiro. Av. Carlos Chagas Filho 373/CCS/Bloco J/20 andar, Ilha do Fundão. 21941-902 Rio de Janeiro RJ Brasil.belfortgabriella@ hotmailcom

\begin{abstract}
This study aimed to identify the determinants of low birth weight ( $L B W$ ) amongst children of adolescent mothers through a hierarchical approach in a cross-sectional study of 751 adolescents attended at a public hospital in Rio de Janeiro. Sociodemographic data, prenatal care, and biological and maternal obstetric conditions were analyzed. Possible determinants of $L B W$ were identified in the bivariate analysis and then hierarchical logistic regression models were tested, considering as taggered hierarchy of distal, intermediate, and proximal levels. Variables with $p<0.05$ at each level of analysis were kept in the model, and the adjusted odds ratio (OR) and $95 \%$ confidence interval (CI) were estimated. The prevalence of low birth weight was 10\%. The determinants of $L B W$ were: distal level - non-acceptance of pregnancy (OR $=10.19,95 \% \mathrm{CI}=$ 1.09 to 39.53); intermediate level - having fewer than six prenatal consultations ( $O R=4.29 ; 95 \%$ $C I=1.55$ to 11.83) and not having standardized nutritional care $(\mathrm{OR}=3.18 ; 95 \% \mathrm{CI}=1.18$ to 8.55); and proximal level - preterm delivery (OR $=10.19,95 \% C I=2.12$ to 49.01$)$. The determinants of $L B W$ were maternal characteristics, prenatal care, and birth conditions, which contain certain modifiable social characteristics.
\end{abstract}

Key words Newborn Low birth weight, Teenage pregnancy, Prenatal care
Resumo Objetivou-se identificar os determinantes do baixo peso ao nascer - BPN, em filhos de adolescentes por meio de abordagem hierarquizada. Estudo transversal com 751 adolescentes atendidas em maternidade pública do Rio de Janeiro. Foram analisados dados sociodemográficos, da assistência pré-natal, condições biológicas e obstétricas maternas. Os possiveis determinantes do BPN foram identificados na análise bivariada e a seguir foram testados modelos de regressão logística hierarquizada, considerando o escalonamento hierarquizado em niveis distal, intermediário $e$ proximal. Permaneceram no modelo as variáveis com valor de $\mathrm{p}<0,05$ em cada nível de análise $e$ estimou-se as odds ratio (OR) ajustadas e intervalos de confiança (IC) de 95\%. A prevalência de BPN foi de $10 \%$. Os determinantes do BPN foram: nível distal - a não aceitação da gravidez $(\mathrm{OR}=10,19$; IC 95\% = 1,09-39,53); nível intermediário = ter menos de seis consultas de pré-natal $(O R=4,29 ;$ IC 95\% = 1,55-11,83), não ter assistência nutricional padronizada $(O R=3,18$; IC 95\% = 1,18-8,55); nível proximal - parto prematuro $(\mathrm{OR}=10,19 ;$ IC $95 \%=2,12-49,01)$. $O$ s determinantes do BPN foram características maternas, da assistência pré-natal e condições ao nascer e, dentre estes fatores, temos características sociais modificáveis.

Palavras-chave Recém-nascido de baixo peso, Gravidez na adolescência, Cuidado pré-natal 


\section{Introdução}

De acordo com a Organização Mundial da Saúde $(\mathrm{OMS})^{1}$, o baixo peso ao nascer (BPN), definido como todo nascido vivo com peso menor de 2,500 gramas no momento do nascimento, é fator determinante da mortalidade e morbidade fetal e neonatal, do déficit no desenvolvimento cognitivo e do aumento no risco de doenças crônicas não transmissíveis na vida adulta ${ }^{2}$.

Estimativas globais indicam prevalência de BPN da ordem de $15 \%$, sendo $96,5 \%$ dos casos ocorridos em países em desenvolvimento, especialmente entre a população mais vulnerável ${ }^{1}$. As principais causas desse desfecho são o nascimento pré-termo, o retardo do crescimento intrauterino e a desnutrição fetal ${ }^{1}$. No Brasil, a prevalência de recém-nascidos de baixo peso sofre variações regionais importantes. Em 2013, dentre os nascidos vivos de mães adolescentes brasileiras, 9,5\% apresentaram BPN e no município do Rio de Janeiro este percentual foi de $10,1 \%{ }^{3}$.

As condições ao nascimento resultam de uma complexa relação entre fatores de várias dimensões. Estudos têm se detido especialmente na relação entre desfechos indesejáveis da gestação e condições genéticas e constitucionais, demográficas e socioeconômicas, nutricionais, obstétricas e de atenção pré-natal ${ }^{1,4-6}$. Em filhos de adolescentes, por exemplo, o BPN tem sido associado com baixa renda e condições de moradia inadequadas, em geral aferidas pelo tipo de habitação e acesso à rede geral de saneamento básico ${ }^{6}$. Tais condições não são diretamente responsáveis pela ocorrência do desfecho, mas contribuem para a influência de alguns determinantes. Dessa forma, o BPN em filhos de adolescentes demanda a proposição de modelos hierarquizados complexos para o estudo de seus determinantes e inter-relações.

Monteiro et al. ${ }^{7}$ apresentam um modelo de determinação do BPN no qual inter-relacionam, de forma hierarquizada, os potenciais fatores de risco já conhecidos na literatura. No primeiro nível desse modelo aparecem as variáveis duração da gestação e velocidade de crescimento intrauterino (determinantes proximais); em segundo nível, estado nutricional da gestante ao início e durante a gravidez, doenças, tabagismo, estresse durante a gestação, adequação do pré-natal, idade materna e paridade (determinantes intermediários). E, no terceiro nível, os condicionantes socioeconômicos renda e escolaridade materna (determinantes distais).

Nascimento ${ }^{8}$, em estudo realizado com mulheres que deram à luz no Hospital Universitário de Taubaté (SP), apresenta como determinantes proximais as variáveis gestacionais - hipertensão gestacional, sangramento vaginal, mesmo não especificado em qual trimestre ocorreu, e ganho de peso insuficiente durante a gestação; como determinantes intermediários os fatores demográficos e reprodutivos - gestação na adolescência, presença de outros filhos com baixo peso e abortamento prévio -; e, como determinantes distais os fatores socioeconômicos - renda familiar e baixa escolaridade materna.

A análise hierarquizada vem sendo utilizada em estudos epidemiológicos nacionais visando elucidar os fatores de risco associados aos agravos relacionados à saúde materno-infantil ${ }^{9}$. Tal análise incorpora níveis hierárquicos diferenciados de determinação em relação a um determinado desfecho ${ }^{10}$. Nesse modelo, os determinantes distais (fatores ambientais e sociodemográficos) influenciam os intermediários (fatores comportamentais e de saúde/doença) e estes, por sua vez, os proximais ${ }^{10}$. Desta forma, a complexidade do desfecho é possível de ser observada, tanto por meio da força da influência que cada um dos fatores exerce sobre sua ocorrência, quanto pelas inter-relações e interdependências desses diferentes fatores no desencadeamento de episódios que favorecem o desenvolvimento do desfecho ${ }^{10}$.

Esse tipo de análise permite identificar a influência de determinantes sociais da saúde, definidos como as condições sociais de vida e trabalho, nas quais as pessoas estão inseridas, e que são conformadas por "fatores sociais, econômicos, culturais, étnicos/raciais, psicológicos e comportamentais, sobre a ocorrência de problemas de saúde e seus fatores de risco na população" ${ }^{11}$, determinantes esses ainda pouco investigados nos estudos brasileiros. Quando avaliados em alguns tipos de análises, os determinantes sociais de saúde não conseguem ser destacados por apresentarem menor força que variáveis biológicas. Contudo, a análise hierarquizada permite o conhecimento das inter-relações e efeitos de mediação entre estes determinantes e entre aqueles tradicionalmente conhecidos implicados na ocorrência do BPN em filhos de mães adolescentes, o que possibilita a identificação do momento em que exercem maior impacto sobre esse agravo'. Dessa forma, torna-se possível demonstrar a relação entre as desigualdades e iniquidades sociais na saúde perinatal.

$\mathrm{Na}$ presente pesquisa utilizou-se a modelagem hierarquizada para identificar os fatores que determinam a ocorrência do baixo peso ao nascer em filhos de adolescentes, atendidas em maternidade pública do Rio de Janeiro. 


\section{Métodos}

\section{Desenho do estudo}

O presente estudo, do tipo transversal, foi realizado em uma maternidade pública do município do Rio de Janeiro com o banco de dados das pesquisas Programa de acompanhamento nutricional pré-natal para gestantes adolescentes e Ganho de peso gestacional em adolescentes associado ao melhor resultado perinatal, desenvolvido sob a responsabilidade do Grupo de Pesquisa em Saúde Materna e Infantil (GPSMI) do Instituto de Nutrição Josué de Castro (INJC) da Universidade Federal do Rio de Janeiro (UFRJ). A referida maternidade é referência para atendimento de gestantes adolescentes e a taxa de acolhimento no pré-natal para estas, com idade $<19$ anos, é em torno de 17,4\% (base em maio/2015; http://www. maternidade.ufrj.br/portal/ images/stories/pdfs/ indicadores/2015/indicadores_geral_maio.pdf).

\section{População estudada e critérios de inclusão}

A população estudada foi constituída por gestantes adolescentes que receberam a assistência pré-natal (APN), o parto e o puerpério na maternidade estudada durante o período de 2004 a 2010 e no ano de 2013. Adotou-se como critérios de seleção da amostra para a presente casuística: ter idade menor que 20 anos na concepção, ter recebido APN, gestação de feto único, ausência de doenças crônicas e ter disponibilidade da informação sobre o peso ao nascer nos prontuários.

\section{Cálculo do tamanho amostral}

Como o número de mulheres com informações disponíveis para esta análise foi menor do que no total da amostra do estudo original, foram realizados cálculos do tamanho da amostra post-hoc. Levando-se em conta uma prevalência de $10 \%$ de BPN e um nível de significância de 5\%, na amostra de em torno de 700 mulheres, com um poder de $80 \%$ para detectar diferenças de pelo menos $6 \%$ na prevalência de BPN entre os grupos (GI/GIII e GII), a amostra estimada mínima para o presente estudo seria de 530 mulheres.

\section{Coleta de dados}

A coleta de dados, realizada por equipe treinada e supervisionada, ocorreu por meio de consulta aos prontuários das adolescentes e recémnascidos e por entrevista realizada durante as consultas de nutrição no pré-natal.
A variável dependente (baixo peso ao nascer) foi classificada segundo a definição da WHO (peso ao nascer $<2,500 \mathrm{~g})^{1}$. As variáveis independentes estudadas foram: dados sociodemográficos, da assistência pré-natal e nutricional prénatal, e características biológicas e obstétricas maternas. Com base na revisão de literatura ${ }^{4,12-14}$, foi proposto modelo teórico conceitual hierarquizado.

\section{Avaliação antropométrica materna e de intercorrências gestacionais}

A avaliação antropométrica foi realizada a partir do peso pré-gestacional ou medido até a $13^{a}$ semana gestacional, estatura e peso pré-parto ou da última consulta do pré-natal. O estado nutricional foi determinado a partir do índice de massa corporal (IMC) pré-gestacional, segundo a recomendação da WHO para indivíduos de 5 a 19 anos, de acordo com o sexo e a idade em meses, por meio da qual se encontrou o percentil referente ao IMC pré-gestacional ${ }^{15}$. Em seguida, obteve-se a classificação do estado nutricional, em baixo peso ou normal ou sobrepeso ou obesidade, de acordo com os padrões ${ }^{15}$ do Sistema de Vigilância Alimentar e Nutricional, propostos pelo Ministério da Saúde ${ }^{16}$. O ganho de peso gestacional foi calculado e avaliado segundo as recomendações do Institute of Medicine ${ }^{17}$.

Com base nas recomendações do Ministério da Saúde ${ }^{18}$, as intercorrências gestacionais estudadas foram: anemia (hemoglobina $<11 \mathrm{~g} / \mathrm{dl}$ ) e síndromes hipertensivas da gravidez (hipertensão gestacional, pré-eclâmpsia, eclampsia). Investigou-se ainda a presença de cegueira noturna gestacional e diabetes gestacional, de acordo com critérios específicos ${ }^{19-21}$, e a presença de qualquer outra intercorrência na gravidez. Em relação aos recém-nascidos, avaliou-se peso e idade gestacional ao nascer.

\section{Modelo hierarquizado}

No presente estudo, determinou-se as variáveis de interesse a partir do conhecimento vigente sobre BPN e, a seguir, adotou-se a classificação dos níveis hierárquicos clássica ${ }^{10}$, na qual o modelo é estruturado com três níveis de hierarquia: distal, intermediário e proximal, visando discriminar as relações entre os determinantes e destes com o BPN em filhos de adolescentes (Figura 1).

Considerou-se como condição de saneamento da moradia adequada quando existisse disponibilidade de água encanada, coleta de lixo e tra- 


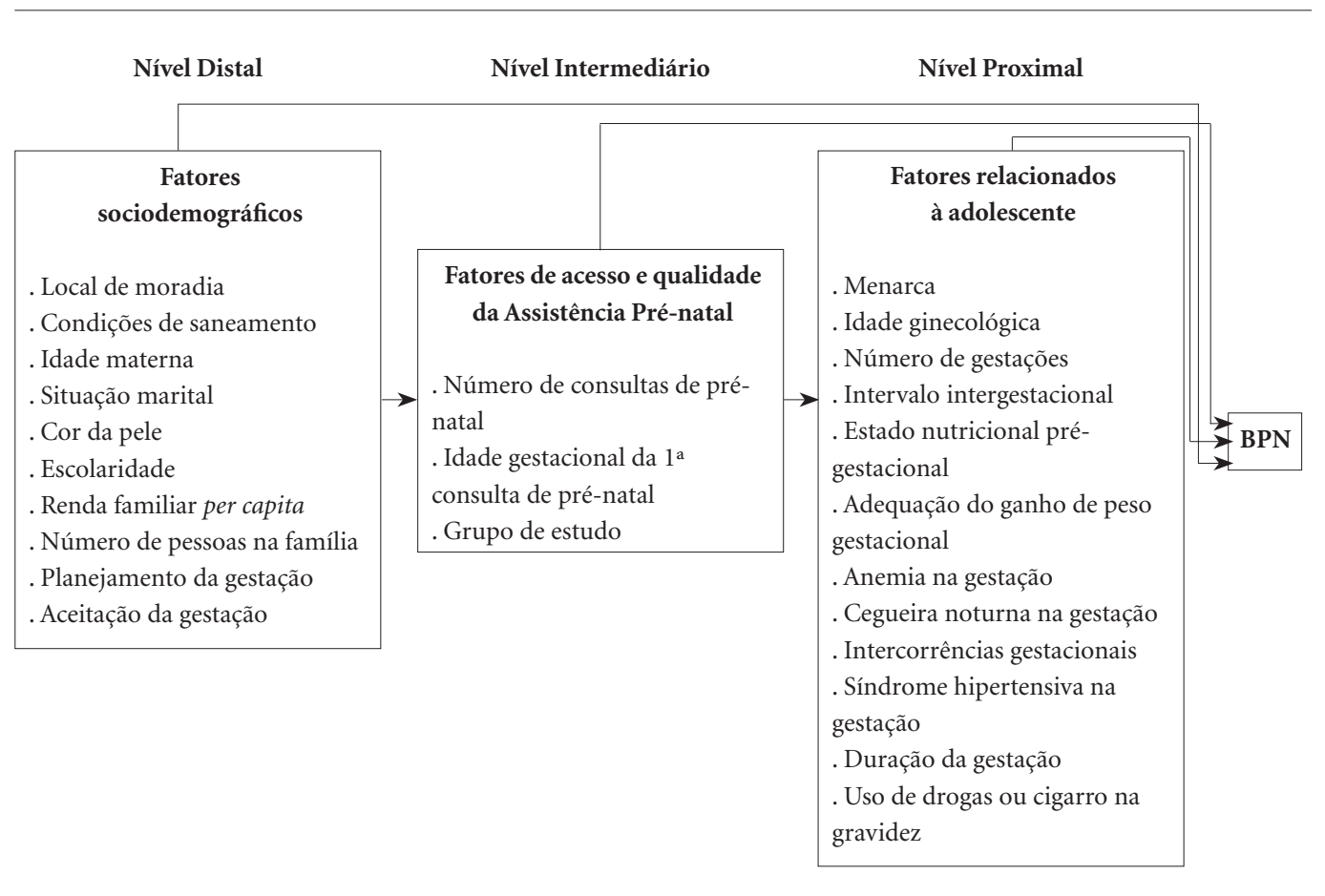

Figura 1. Descrição das variáveis independentes segundo o modelo hierarquizado. Rio de Janeiro/ RJ, 2013.

tamento de esgoto e como inadequada, quando um destes serviços estivesse ausente ${ }^{22}$.

A variável grupo de estudo foi criada para representar a assistência nutricional recebida pelas gestantes durante o pré-natal e a categorização ocorreu em função do tipo de assistência nutricional oferecida. O grupo II (GII - 2007-2010) recebeu a assistência nutricional diferenciada, caracterizada por: início precoce do acompanhamento com o nutricionista no pré-natal e realização de no mínimo 4 consultas com o este, intercaladas com consultas em grupo, onde eram realizadas ações de vigilância e prevenção de intercorrências clínicas e avaliação nutricional individualizada. Os grupos I (GI - 2004-2006) e III (GIII - 2013) foram encaminhados ao nutricionista em qualquer idade gestacional e não foi obedecido o calendário mínimo de 4 consultas.

Em relação ao estado nutricional pré-gestacional, a categorização das variáveis ocorreu conforme o IMC pré-gestacional - $\mathrm{kg} / \mathrm{m}^{2}$ (baixo peso - IMC < percentil - p 3/ normal - IMC entre p 3 e $<$ p 85, sobrepeso - IMC entre p 85 e $<$ p 97 e obesidade - IMC $\geq$ p 97). Optou-se por avaliar o baixo peso em comparação às demais classificações uma vez que o baixo peso pré-gestacional pode se relacionar com o $\mathrm{BPN}^{23}$.

\section{Análise de dados}

A associação entre os possíveis determinantes do BPN com a gravidez na adolescência foi avaliada por meio de análise bivariada, com todas as variáveis de cada nível hierárquico. Foram estimadas as odds ratio (OR) brutas com os intervalos de confiança (IC) de 95\%, por meio da regressão logística simples. Para elaboração do modelo hierarquizado final, as variáveis foram introduzidas no modelo considerando os níveis distal, intermediário e proximal. Como critério de inclusão das variáveis no modelo foi considerado o valor de $p<0,20$ obtido na análise bivariada. Para o ajuste do modelo em nível de hierarquia, permaneceram no modelo as variáveis com valor de $p<0,05$ em cada nível de análise.

No modelo final foram estimadas as OR ajustadas com seus respectivos IC de 95\%, por meio da regressão logística hierarquizada e de acordo com cada nível de hierarquia. Foram realizados os testes Qui-quadrado, para avaliar associação entre determinantes do BPN e características da amostra, e o teste t-Student para a comparação de médias. As análises foram realizadas com auxílio do programa estatístico SPSS (Statistical Package for Social Sciences) versão 21.0. 


\section{Questões éticas}

O estudo foi elaborado atendendo os aspectos éticos previstos nas resoluções 196/96 e 466/2012 do Conselho Nacional de Saúde e aprovado pelo Comitê de Ética em Pesquisa da Maternidade Escola da Universidade Federal do Rio de Janeiro. Apenas as participantes do grupo III assinaram o Termo de Consentimento Livre e Esclarecido, pois os dados dos demais grupos foram coletados exclusivamente via prontuário e o pesquisador se responsabilizou pela confiabilidade dos dados.

\section{Resultados}

Dez por cento dos recém-nascidos apresentaram BPN e 13\% tiveram nascimento prematuro. Foram incluídas no estudo 751 adolescentes e seus respectivos filhos. De acordo com a população do estudo original $(n=845)$ ocorreram 94 perdas, sendo $37 \%$ casos de aborto e $59 \%$ por falta da informação do peso ao nascer. A análise comparativa entre as adolescentes consideradas como perdas e as incluídas na pesquisa apontou a inexistência de diferença estatística $(p>0,05)$ em relação às variáveis idade materna no parto, escolaridade, aceitação da gravidez pela gestante e número de consultas de APN.

As características sociodemográficas das participantes revelaram que a média de idade no parto foi de 17,5 anos $\pm 1,6$, que $64 \%$ possuíam ensino fundamental completo, $52 \%$ não trabalhavam, $68 \%$ eram solteiras, $62 \%$ se autodeclararam como não brancas, $64 \%$ apresentaram renda per capita inferior a um salário mínimo, 52\% eram moradoras da zona sul do Rio de Janeiro e 89\% tinham acesso a saneamento básico adequado Embora o planejamento da gravidez tenha ocorrido para $22 \%$ das adolescentes, a aceitação da gestação foi relatada por $95 \%$.

Setenta e dois por cento das adolescentes tiveram seis ou mais consultas de pré-natal e $80 \%$ receberam assistência nutricional pré-natal (ANPN). Oitenta e um por cento das jovens iniciaram a gravidez com estado nutricional adequado, todavia, $66 \%$ apresentaram inadequação no ganho de peso recomendado. Vinte e seis por cento teve alguma intercorrência gestacional, sendo a anemia o agravo mais frequente. Dentre as síndromes hipertensivas da gravidez, a mais incidente foi a hipertensão gestacional $(n=20)$, enquanto que a cegueira noturna foi relatada por 36 adolescentes.
Por meio da análise bivariada identificou-se os fatores associados com o BPN ( $p<0,20)$, nos três níveis de hierarquia. A Tabela 1 apresenta a associação entre as dimensões que compõem o nível referente às características socioeconômicas (nível distal) e o BPN. Verificou-se que as varáveis idade materna, situação marital, aceitação da gravidez e escolaridade associaram-se ao BPN. Destacaram-se como contribuintes da ocorrência de BPN neste nível, a não aceitação da gravi$\operatorname{dez}(\mathrm{OR}=5,7 ;$ IC $95 \%=1,67-19,84)$ e a ausência de companheiro (OR = 2,28; IC 95\% = 1,045,02). Identificou-se associação entre a aceitação da gravidez com o planejamento de engravidar e com o número de consultas de APN inferior a seis $(p=0,01)$. Observou-se ainda o início mais tardio da APN entre aquelas que não aceitaram a gestação (média $=17,6$ semanas gestacionais versus 16 semanas gestacionais entre as que aceitaram a gravidez) e que a não aceitação foi mais frequente nas adolescentes menores de 16 anos (6,7\%; versus 5,1\% maiores de 16 anos).

Quanto às características da APN (nível intermediário), as que se associaram ao desfecho foram: número de consultas de assistência prénatal e grupo de estudo (Tabela 2). O número de consultas de APN inferior a seis se relacionou ao menor grau de escolaridade $(\mathrm{p}<0,001)$.

Para as características biológicas e obstétricas (nível proximal) associaram-se ao desfecho: sexarca, estado nutricional pré-gestacional, adequação do ganho de peso gestacional e duração da gestação (Tabela 3). O fator de maior impacto deste nível foi a duração da gestação $(\mathrm{OR}=36,6$; IC $95 \%=19,60-68,55)$, seguido da adequação do ganho de peso gestacional $(\mathrm{OR}=2,94$; IC 95\% $=1,39-6,24)$. Foi identificado, por meio do teste $t$-Student, que as adolescentes representantes dos GI e GIII apresentaram menor número médio de consultas com o nutricionista $(2,6 \pm 1,6$ consultas versus $3,8 \pm 1,7$ consultas para participantes do grupo II; $\mathrm{p}<0,001)$.

No modelo final, após os ajustes, foi observado que não aceitar a gravidez (nível distal, OR ajustada $=6,56$; IC 95\% = 1,09 - 39,53), possuir menos de seis consultas de assistência pré-natal (nivel intermediário, OR ajustada = 4,29; IC 95\% $=1,56-11,83)$, pertencer ao grupo de estudo I ou III (nível intermediário, OR ajustada $=3,18$; IC 95\% = 1,18 - 8,55) e ter duração da gestação inferior a 37 semanas, (nível proximal, OR ajustada $=10,19 ;$ IC $95 \%=2,12-49,01)$ foram os determinantes do BPN (Tabela 4). 
Tabela 1. Fatores determinantes sociodemográficos (distais) do baixo peso ao nascer nos filhos de adolescentes atendidas em maternidade pública do Rio de Janeiro. Rio de Janeiro, RJ, Brasil (2004-2010 e 2013).

\begin{tabular}{|c|c|c|c|c|}
\hline Variáveis & $\underset{\mathbf{n}}{\text { Amostra }}$ & $\begin{array}{c}\text { Baixo peso ao nascer } \\
\mathbf{n}(\%)\end{array}$ & $\mathbf{p}^{\mathrm{a}}$ & OR (IC95\%) \\
\hline Local de moradia & 750 & & & \\
\hline Não mora na Zona Sul & 361 & $38(10,5)$ & 0,64 & $1,12(0,70-1,81)$ \\
\hline Mora na Zona Sul & 389 & $37(9,5)$ & & 1 \\
\hline Condições de saneamento da moradia & 458 & & & \\
\hline Inadequada & 48 & $4 \quad(8,3)$ & 0,75 & $1,19(0,40-3,56)$ \\
\hline Adequada & 410 & $29(7,1)$ & & 1 \\
\hline Idade materna & 749 & & & \\
\hline$<16$ anos & 112 & $16(14,3)$ & 0,10 & $1,63(0,90-2,95)$ \\
\hline$\geq 16$ anos & 637 & $59(9,3)$ & & 1 \\
\hline Situação marital & 538 & & & \\
\hline Vive sem companheiro & 367 & $37(10,1)$ & 0,04 & $2,28(1,04-5,02)$ \\
\hline Vive com companheiro & 171 & $8(4,7)$ & & 1 \\
\hline Cor da pele & 586 & & & \\
\hline Não branca & 363 & $33(9,1)$ & 0,81 & $1,07(0,59-1,94)$ \\
\hline Branca & 223 & $19(8,5)$ & & 1 \\
\hline Escolaridade & 662 & & & \\
\hline Fundamental incompleto & 240 & $32(13,3)$ & 0,02 & $1,87(1,12-3,15)$ \\
\hline Fundamental completo & 422 & $32(7,6)$ & & 1 \\
\hline Renda familiar per capita & 201 & & & \\
\hline$<1$ salário mínimo & 129 & $8(6,2)$ & 0,85 & $1,12(0,33-3,87)$ \\
\hline$\geq 1$ salário mínimo & 72 & $4(5,6)$ & & 1 \\
\hline Número de pessoas da familia & 254 & & & \\
\hline$>4$ & 70 & $6(8,6)$ & 0,46 & $1,47(0,52-4,15)$ \\
\hline$\leq 4$ & 184 & $11(6,0)$ & & 1 \\
\hline Planejamento da gestação & 385 & & & \\
\hline Não & 300 & $21(7,0)$ & & $1,20(0,44-3,29)$ \\
\hline Sim & 85 & $5(5,9)$ & 0,72 & 1 \\
\hline Aceitação da gravidez pela gestante & 308 & & & \\
\hline Não & 16 & $4(25,0)$ & & $5,70(1,67-19,84)$ \\
\hline Sim & 292 & $16(5,5)$ & 0,01 & 1 \\
\hline
\end{tabular}

Legenda: OR = odds ratio; IC 95\% = intervalo de confiança de 95\%; ${ }^{a}$ regressão logística bivariada.

\section{Discussão}

A prevalência de BPN neste estudo foi de $10 \%$. Este indicador apresenta grande variação entre as regiões brasileiras, uma vez que pesquisas com gestantes menores de 20 anos realizadas no Sudeste e no Nordeste do país identificaram prevalências de $15,1 \%$ e $11,9 \%$, respectivamente ${ }^{24,25}$. Lima et al. ${ }^{26}$ ao estudarem a variabilidade dessa prevalência no Brasil, apontaram que a taxa de BPN está relacionada ao meio social em que se vive e à desigualdade de acesso aos serviços de saúde. De acordo com os autores, a falta de recursos na assistência médica hospitalar e de registros de peso ao nascer em regiões menos desenvolvidas contribui para que estas apresentem menores taxas, em contraste às regiões mais desenvolvidas que apresentam APN de melhor qualidade, favorecendo menor taxa de mortalidade infantil e mais registros do peso ao nascer.

Ganchimeg et al. ${ }^{27}$, em pesquisa realizada em países de baixo e médio desenvolvimento encontraram maior prevalência de BPN, igual a 12,3\%, entre filhos de adolescentes. Este fato pode estar relacionado às piores condições sociodemográficas e de APN de alguns países da África e Ásia incluídos no estudo, que apresentavam maior número de adolescentes com baixo IMC pré-gestacional e piores níveis de escolaridade, quando comparado aos países da América Latina também avaliados. Entretanto, a taxa de BPN deste estudo ficou no limiar da recomendação da Or- 
Tabela 2. Associação entre características da assistência pré-natal (intermediárias) e baixo peso ao nascer de filhos de adolescentes atendidas em maternidade escola do Rio de Janeiro, RJ, Brasil (2004-2010 e 2013).

\begin{tabular}{|c|c|c|c|c|}
\hline Variáveis & $\begin{array}{c}\text { Amostra } \\
\mathbf{n}\end{array}$ & $\begin{array}{c}\text { Baixo Peso ao nascer } \\
\text { n (\%) }\end{array}$ & $\mathbf{p}^{\mathrm{a}}$ & OR (IC95\%) \\
\hline Número de consultas de pré-natal & 679 & & & \\
\hline$<6$ & 192 & $33(17,2)$ & $<0,001$ & $3,40(1,99-5,81)$ \\
\hline$\geq 6$ & 487 & $28(5,7)$ & & 1 \\
\hline Idade gestacional na $1^{\text {a }}$ consulta de APN & 517 & & & \\
\hline$\leq 16$ & 200 & $18(9,0)$ & 0,95 & $1,02(0,55-1,90)$ \\
\hline$>16$ & 317 & $28(8,8)$ & & 1 \\
\hline Grupos de estudo & 751 & & & \\
\hline GI e GIII & 555 & $66(11,9)$ & $<0,001$ & $2,80(1,37-5,74)$ \\
\hline GII & 196 & $9(4,6)$ & & 1 \\
\hline
\end{tabular}

ganização das Nações Unidas, que propõe que a prevalência de crianças com BPN não ultrapasse $10 \%{ }^{28}$. De acordo com a $\mathrm{WHO}^{1}$, no ano de 2000 a prevalência de BPN em países desenvolvidos ficava em torno de $7 \%$ e na América do Sul em torno de 9,6\%. Em 2014 esta taxa na América Latina foi de $9 \%{ }^{2}$, refletindo a dificuldade de controle sobre este desfecho negativo.

A aplicação do modelo hierarquizado na presente investigação apontou que, dentre os fatores sociodemográficos analisados, a não aceitação da gravidez (nível distal) se revelou como determinante do BPN. Este achado pode relacionar-se com o planejamento da gestação, pois verificouse que todas aquelas que não aceitaram a gestação foram adolescentes que não a planejaram, diferindo do grupo que realizou planejamento. Outro aspecto que pode ser atribuído à aceitação da gravidez é a idade materna, que se relaciona com a imaturidade psicológica. No presente estudo, aquelas que não aceitaram a gravidez eram, em maior proporção, menores de 16 anos.

Entretanto, Moreira et al. $^{29}$, ao estudarem grávidas adolescentes brasileiras, encontraram outros determinantes para a não aceitação da gravidez, como: a reação negativa dos pais, negação de apoio à gestante, e baixo nível socioeconômico, relacionado às baixas condições de saúde e de educação, corroborando para ocorrência da gravidez indesejada.

Phipps e Nunes $^{30}$, que avaliaram a associação da intenção de engravidar com riscos à saúde materno-infantil, encontraram que a falta de preparo emocional da adolescente na concepção esteve associada com a inadequação do cuidado pré-natal $(\mathrm{OR}=2,7$; IC 95\% = 1,27 - 5,72). E, de acordo com meta-análise de Shah et al. ${ }^{31}$, a gravidez indesejada pode aumentar em 1,4 vezes $(\mathrm{OR}=1,36$; IC $95 \%=1,25-1,48)$ a chance de ocorrer o BPN.

A não aceitação da gravidez nesta pesquisa associou-se ao início da APN mais tardio e ao número de consultas inferior a seis, confirmando os achados de outras pesquisas que sugerem que a gravidez indesejada é associada ao cuidado prénatal inadequado ${ }^{32}$.

O início tardio e o menor número de consultas de APN são características reconhecidas desta população ${ }^{33}$ e estão associadas a desfechos perinatais negativos como a prematuridade e o $\mathrm{BPN}^{8}$. Pesquisa canadense verificou que as jovens, além do início tardio do pré-natal, apresentaram piores condições de saúde, menor uso de ácido fólico, menores taxas de início e duração de aleitamento materno e filhos com a saúde inferior quando comparadas às adultas jovens ${ }^{34}$.

Segundo diretrizes do Ministério da Saúde do Brasil, a APN deve garantir a oferta de no mínimo seis consultas de pré-natal, como condição para assegurar bem-estar materno e neonatal ${ }^{17}$, condizendo com o presente achado. Realizar menos de seis consultas de APN foi determinante para o BPN entre os filhos das adolescentes, assim como observado no estudo de Santos et al. ${ }^{12}(\mathrm{OR}=2,7$; IC $95 \%=1,48-5,05)$. Outra pesquisa realizada com gestantes adolescentes e adultas, que propôs um modelo hierarquizado para o BPN, encontrou que o menor número de consultas de prénatal ( $<6$ - nível intermediário) aumentou em 1,7 vezes a chance de ocorrer o $\mathrm{BPN}^{25}$.

No presente estudo observou-se ainda que o menor número de consultas de APN esteve associado à menor escolaridade e ressalta-se que na análise bivariada a escolaridade apresentou asso- 
Tabela 3. Associação entre características maternas proximais (biológicas, obstétricas e clínicas - proximais) e baixo peso ao nascer de filhos de adolescentes atendidas em maternidade escola do Rio de Janeiro, RJ, Brasil (2004-2010 e 2013).

\begin{tabular}{|c|c|c|c|c|}
\hline Variáveis & $\begin{array}{c}\text { Amostra } \\
\mathbf{n}\end{array}$ & $\begin{array}{c}\text { Baixo Peso ao nascer } \\
\mathbf{n}(\%)\end{array}$ & $\mathrm{p}^{\mathrm{a}}$ & OR (IC95\%) \\
\hline Menarca & 347 & & & \\
\hline$<13$ anos & 222 & $19(8,6)$ & 0,47 & $1,37(0,58-3,22)$ \\
\hline$\geq 13$ anos & 125 & $8(6,4)$ & & 1 \\
\hline Idade ginecológica & 421 & & & \\
\hline$\leq 2$ anos & 60 & $5(8,3)$ & 0,76 & $1,17(0,43-3,18)$ \\
\hline$>2$ anos & 361 & $26(7,2)$ & & 1 \\
\hline Número de gestações & 751 & & & \\
\hline Não primigesta & 171 & $21(12,3)$ & 0,26 & $1,36(0,80-2,33)$ \\
\hline Primigesta & 580 & $54(9,3)$ & & 1 \\
\hline Intervalo intergestacional & 70 & & & \\
\hline$<24$ meses & 47 & $5(10,6)$ & 0,80 & $1,25(0,22-6,99)$ \\
\hline$\geq 24$ meses & 23 & $2(8,7)$ & & 1 \\
\hline Estado nutricional pré-gestacional & 452 & & & \\
\hline Baixo peso & 62 & $8(12,9)$ & 0,09 & $2,70(0,42-2,46)$ \\
\hline Normal/ Sobrepeso /Obesidade & 390 & $26(6,7)$ & & 1 \\
\hline Adequação do ganho de peso total & 409 & & & \\
\hline Abaixo & 122 & $16(13,1)$ & $<0,001$ & $2,94(1,39-6,24)$ \\
\hline Adequado ou acima & 287 & $14(4,9)$ & & 1 \\
\hline Anemia na gestação & 569 & & & \\
\hline $\operatorname{Sim}$ & 234 & $21(11,5)$ & 0,44 & $1,23(0,72-2,12)$ \\
\hline Não & 335 & $32(9,6)$ & & 1 \\
\hline Cegueira noturna gestacional & 330 & & & \\
\hline Sim & 36 & $4(11,1)$ & 0,22 & $2,04(0,65-6,43)$ \\
\hline Não & 294 & $17(5,8)$ & & 1 \\
\hline Intercorrências na gestação & 715 & & & \\
\hline Com intercorrências & 188 & $23(12,2)$ & 0,25 & $1,36(0,80-2.30)$ \\
\hline Sem intercorrências & 527 & $49(9,3)$ & & 1 \\
\hline SHG & 751 & & & \\
\hline Não & 719 & $72(10,0)$ & 0,91 & $1,08(0,32-3,62)$ \\
\hline Sim & 32 & $3(9,4)$ & & 1 \\
\hline Duração da gestação & 667 & & & \\
\hline$<37$ semanas & 85 & $47(55,3)$ & $<0,001$ & $36,6(19,60-68,54)$ \\
\hline$\geq 37$ semanas & 582 & $19(3,3)$ & & 1 \\
\hline Uso de cigarro durante a gravidez & 448 & & & \\
\hline Sim & 43 & $5(11,6)$ & 0,30 & $1,71(0,62-4,66)$ \\
\hline Não & 405 & $29(7,2)$ & & 1 \\
\hline Uso de drogas durante a gravidez & 449 & & & \\
\hline Sim & 12 & $1(8,3)$ & 0,94 & $1,08(0,13-8,60)$ \\
\hline Não & 437 & $34(7.8)$ & & 1 \\
\hline
\end{tabular}

ciação com o BPN, confirmando uma influência deste indicador social (nível distal) sobre características de assistência pré-natal (nível intermediário). Segundo Viner et al. ${ }^{35} \mathrm{o}$ acesso à educação representa um forte determinante social da saúde dos adolescentes, sendo a escola uma instituição crucial para apoiar o processo de amadurecimen- to e desenvolvimento biopssicosocial para que o jovem possa realizar uma transição saudável para a vida adulta.

Todavia, embora a escola seja um espaço privilegiado para o exercício da socialização e transmissão de normas, valores e conhecimentos, é sabido que isso não ocorre de forma igualitária, 
Tabela 4. Modelo final hierarquizado com as OR brutas e ajustadas para estimativa dos determinantes do baixo peso ao nascer em filhos de adolescentes atendidas em maternidade pública do Rio de Janeiro. Rio de Janeiro, RJ, Brasil (2004-2010 e 2013).

\begin{tabular}{|c|c|c|c|c|c|c|c|}
\hline & & $p^{c}$ & $\begin{array}{c}\text { OR } \\
\text { bruta }\end{array}$ & IC $95 \%$ & $p^{c}$ & $\begin{array}{c}\text { OR } \\
\text { ajustada }\end{array}$ & IC $95 \%$ \\
\hline \multicolumn{8}{|l|}{ Modelo Distal } \\
\hline \multirow{2}{*}{$\begin{array}{l}\text { Aceitação da gravidez } \\
\text { pela gestante }\end{array}$} & Não & 0,01 & 5,70 & $1,67-19,84$ & 0,04 & 6,56 & $1,09-39,53$ \\
\hline & Sim & $\ldots$ & 1,00 & $\ldots$ & & 1,00 & $\ldots$ \\
\hline \multicolumn{8}{|l|}{ Modelo Intermediário ${ }^{\mathrm{a}}$} \\
\hline \multirow[t]{2}{*}{ No de consultas de PN } & $<6$ & $<0,001$ & 3,40 & $1,99-5,81$ & $<0,001$ & 4,29 & $1,55-11,83$ \\
\hline & $6 \mathrm{ou}+$ & $\ldots$ & 1,00 & $\ldots$ & $\ldots$ & 1,00 & $\ldots$ \\
\hline \multirow[t]{2}{*}{ Grupo de estudo } & GI e GIII & $<0,001$ & 2,80 & $1,37-5,74$ & 0,02 & 3,18 & $1,18-8,55$ \\
\hline & GII & $\ldots$ & 1,00 & $\ldots$ & $\ldots$ & 1,00 & $\ldots$ \\
\hline \multicolumn{8}{|l|}{ Modelo Proximal ${ }^{\mathrm{b}}$} \\
\hline \multirow[t]{2}{*}{ Duração da gestação } & $<37$ semanas & $<0,001$ & 36,6 & $19,60-68,54$ & $<0,001$ & 10,19 & $2,12-49,01$ \\
\hline & $\geq 37$ semanas & $\ldots$ & 1,00 & $\ldots$ & $\ldots$ & 1,00 & $\ldots$ \\
\hline
\end{tabular}

a ajustado por aceitação da gestação pela gestante, ${ }^{\mathrm{b}}$ ajustado por grupo de estudo e número de consultas de pré-natal. ${ }^{\mathrm{c}}$ Regressão logística hierarquizada. Legenda: No. - número; PN - pré-natal; OR = odds ratio; IC 95\% = intervalo de confiança de 95\%.

muitas vezes reforçando as desigualdades sociais, de classe e de gênero. Aliado a isso, encontra-se condições de pobreza e fragilidade familiar que promovem a evasão escolar, aumentando a proporção de adolescentes cujo horizonte de seus projetos de vida restringe-se a um futuro imediato, com pouca expectativa e baixíssima autoesti$\mathrm{ma}^{36}$. Talvez, esses possam constituir alguns dos fatores que determinam a baixa frequência às consultas de pré-natal e seu consequente impacto negativo nos resultados perinatais.

Em relação à cor da pele não foi encontrada associação com o desfecho, embora em pesquisa brasileira com grávidas adultas e adolescentes ${ }^{37}$, observou-se que entre as que possuíam cor da pele preta ocorreu baixa proporção do início do pré-natal e do número de consultas que, como já mencionado, é fator determinante do BPN. De acordo com Meyer et al. ${ }^{38}$, a questão racial parece interferir nos resultados perinatais pelo fato do acesso à educação se relacionar a questão das disparidades raciais, remetendo às mesmas consequências apontadas no parágrafo anterior.

A condição de saneamento também não se apresentou como determinante do BPN. Entretanto, é relatado na literatura que as condições de moradia consideradas ruins, dentre elas o acesso ao saneamento básico, podem influenciar para ocorrência o BPN e prematuridade ${ }^{6}$. Este indicador social de saúde é relacionado à condição de pobreza, que por sua vez pode ser um proxy das condições de saúde, por isso foi selecionado como variável a ser testada no nível distal ${ }^{6}$.
Quanto à assistência nutricional pré-natal, observou-se que esta intervenção, de forma regular, pode contribuir para a redução de BPN, assim como do diabetes gestacional, pré-eclâmpsia e prematuridade, conforme descrito por Vitolo et al. ${ }^{39}$. Em outra pesquisa brasileira, com grávidas adolescentes, verificou-se que a ausência da intervenção nutricional aumentou em 3,5 vezes a chance de $\mathrm{BPN}^{22}$.

A importância da ANPN vem sendo elucidada à medida que estudos demonstram a relevância da adequação do ganho de peso e do consumo alimentar saudável durante a gestação, sobre resultados perinatais. Guerra et al. ${ }^{40}$, ao avaliarem o impacto do estado nutricional de gestantes adolescentes sobre recém-nascidos, encontraram correlação positiva do ganho de peso gestacional com o peso ao nascer $(\mathrm{r}=0,41 ; \mathrm{p}=0,00)$. E, segundo Padilha et al. ${ }^{23}$, o ganho de peso durante a gravidez $(\mathrm{p}=0,00)$, assim como o IMC prégestacional $(\mathrm{p}=0,04)$, são preditores do peso ao nascer.

Entretanto, a inadequação do ganho de peso gestacional, apresentada por adolescentes em alguns estudos, foi observada na maioria das participantes desta pesquisa ${ }^{12,40}$. E de acordo com o último estudo de base populacional do Instituto Brasileiro de Estatística e Geografia ${ }^{41}$ sobre consumo alimentar, a maior parte da população de adolescentes apresenta alto consumo de alimentos ultraprocessados e baixo consumo de alimentos in natura ou aqueles reconhecidos como minimamente processados, produzindo deficiên- 
cias nutricionais principalmente de vitaminas $\mathrm{A}$, E, D, C e dos minerais cálcio, fósforo e magnésio. Classicamente, é reconhecida a associação entre as deficiências de vitamina A e D com o baixo peso ao nascer ${ }^{42,43}$.

A redução do consumo de produtos ultraprocessados (alimentos fabricados por indústrias, considerados nutricionalmente desbalanceados, sendo muitas vezes ricos em açúcar, sódio, gordura e pobres em fibras), objetivando o aumento da ingestão dos alimentos minimamente processados ou in natura (obtidos diretamente da natureza e consumidos sem sofrer nenhuma alteração ou após processamento mínimo, considerados boas fontes de fibras, vitaminas, minerais e proteínas) é recomendada no novo Guia Alimentar para a População Brasileira ${ }^{44}$.

Desse modo, sugere-se que a ANPN exerça proteção contra o $\mathrm{BPN}$, demonstrando que as orientações nutricionais são essenciais para corrigir inadequações alimentares e controlar o ganho de peso, que são fatores associados ao desenvolvimento de uma gestação saudável e à prevenção do desenvolvimento de doenças crônicas não transmissíveis durante a vida da criança. Segundo Barker ${ }^{45}$, a nutrição deficiente durante a gestação e infância precoce originaria uma adaptação metabólica e/ou estrutural permanente no ambiente intrauterino, que aumentaria o risco de desenvolvimento de doença coronária e outras doenças associadas, como hipertensão arterial, diabetes e acidente vascular cerebral, na vida adulta.

Além dos determinantes de ordem social que atuam indiretamente sobre o desfecho, e permitem maiores possibilidades de intervenção, ressalta-se a importância da identificação dos fatores de ordem biológica materna que se relacionam diretamente com o desenvolvimento do desfecho, a fim de obter o maior controle possível sobre o BPN. Na literatura é reconhecido que a duração da gestação inferior a 37 semanas (parto pré-termo) possui relação com o $\mathrm{BPN}^{1}$. E que gestar na adolescência caracteriza-se como risco independente para o nascimento prematuro, podendo este risco ser 1,7 vezes maior em adolescentes menores de 15 anos comparando-se a adultas $^{46}$. Essa predisposição pode ser em virtude da imaturidade biológica materna e ao consequente maior risco para intercorrências gestacionais que favorecem a prematuridade.

Embora a chance de BPN tenha sido 10 vezes maior entre os prematuros, a prematuridade nesta pesquisa parece ter apresentado menor força de determinação sobre a variável dependente quando comparada aos dados da literatura atual. Em pesquisa já citada ${ }^{25}$, realizada no Nordeste brasileiro, para encontrar os determinantes do BPN a partir de um modelo hierarquizado, a prematuridade (nível proximal) aumentou em 21,8 vezes a chance de BPN. Em outro estudo com gestantes adultas e adolescentes da região Sul do Brasil, que buscou identificar os fatores associados ao BPN, verificou-se que a prematuridade aumentava em até 37 vezes a chance de ocorrer o baixo peso ao nascimento ${ }^{13}$. Sugere-se que este resultado encontrado na presente pesquisa seja devido ao fato de a unidade de saúde na qual ocorreu o estudo ser referência na atenção pré-natal para gestantes adolescentes, oferecendo atendimento especializado e, por isso, a maioria das gestantes ter realizado um pré-natal adequado ( $>6$ consultas) e ter recebido algum tipo de ANPN.

A dificuldade para recuperar dados sociodemográficos ausentes no prontuário, tais como renda familiar per capita e número de pessoas na família, foi uma limitação da pesquisa, fazendo com que o quantitativo para essas variáveis tenha sido reduzido, o que se refletiu em IC grandes. Entretanto, apesar do IC maior, observa-se na variável de aceitação da gravidez pela gestante que $1 / 4$ da amostra que não aceitou a gravidez teve filho com BPN e que a variável relacionou-se com o cuidado pré-natal de pior qualidade. Observou-se ainda, que outras características sociodemográficas demonstraram associação com o desfecho no nível distal, reforçando a importância do papel destes fatores. Porém, o preenchimento do prontuário, em relação a estas condições da adolescente por parte dos profissionais de saúde, é precário, sendo uma limitação para o estudo da influência dos determinantes sociais da saúde.

\section{Conclusão}

A análise hierarquizada empregada no presente estudo possibilitou a identificação de um determinante sociodemográfico, que foi a "não aceitação da gravidez", que, por sua vez, pode exercer influências sobre a "menor frequência à APN" e, por conseguinte, "não receber ANPN" e "ter parto prematuro", com estas variáveis atuando sobre o desfecho estudado. Além disso, foi observada a elucidação da associação entre as variáveis estudadas e a identificação do momento em que exercem maior impacto na sua ocorrência.

Sugere-se que os recursos humanos envolvidos com a atenção pré-natal voltada a gestantes adolescentes sejam qualificados, para que possam oferecer suporte emocional e estímulo 
ao apoio familiar para o cuidado da gestante, e para incentivar a promoção do adequado estado nutricional durante a gravidez, por meio da oferta de assistência nutricional diferenciada e de qualidade, com início concomitante ao do prénatal. Destaca-se ainda, a necessidade de ampliar o acesso às informações sobre prevenção e desenvolvimento da gravidez nessa faixa de idade, incluindo a importância da atenção pré-natal e seu início precoce.

\section{Referências}

1. World Health Organization (WHO), United Nations Children's Fund (UNICEF). Low Birth weight: Country, regional and global estimates. New York: UNICEF; 2004.

2. World Health Organization (WHO). Global Nutrition Targets 2025 Low Birth Weight Policy Brief. Geneva. [internet] 2014. [acessado $2015 \mathrm{Fev}$ 10]. Disponível em: http://www.who.int/nutrition/publications/globaltargets2025_policybrief_lbw/en/.

3. Brasil. Ministério da Saúde (MS). Sistema de Informações sobre Nascidos Vivos (SINASC - DATASUS). [internet] 2008 [acessado 2016 Maio 05]. Disponível em: http://tabnet.datasus.gov.br/cgi/tabcgi.exe?sinasc/cnv/ nvuf.def.

4. Fraser AM, Brockert JE, Ward RH. Association of young maternal age with adverse reproductive outcomes. New Engl J Med 1995; 332(17):1113-1117.

5. Kashan AS, Barker PN, Kenny LC. Preterm birth and reduced birth weight in first and second teenage pregnancies: a register-based in cohort study. BMC Pregnancy and Childbirth 2010; 10(36):1-8.

6. Vettore MV, Gama SGN, Lamarca GA, Shilithz AOC, Leal MC. Housing conditions as a social determinant of low birthweight and preterm low birthweight. Rev Saude Publica 2010; 44(6):1021-1031.

7. Monteiro CA, Benicio MHA, Ortiz LP. Tendência secular do peso ao nascer na cidade de São Paulo (19761998). Rev Saude Publica 2000; 34(Supl. 1):26-40.

8. Nascimento LFC. Análise hierarquizada dos fatores de risco para o baixo peso ao nascer. Rev Paul Pediatria 2005; 23(2):76-82.

9. Lima S, Carvalho ML, Vasconcelos AGG. Proposta de modelo hierarquizado aplicado à investigação de fatores de risco de óbito infantil neonatal. Cad Saude Publica 2008; 24(8):1910-1916.

\section{Colaboradores}

GP Belfort, MMAS Santos e C Saunders trabalharam na concepção, desenho do estudo, análise e interpretação dos dados, escrita e revisão do manuscrito. E LS Pessoa, JR Dias e SP Heidelmann contribuíram na escrita e revisão do manuscrito.
10. Mosley WH, Chen LC. An analytical framework for the study of child survival in developing countries. Popul Dev Rev 1984; 10(Supl. 25-45):140-145.

11. Buss PM, Pellegrini Filho A. A saúde e seus determinantes sociais. Phisis 2007; 17(1):77-93.

12. Santos MMAS, Baião MR, Barros DC, Pinto AA, Pedrosa PLM, Saunders C. Estado nutricional pré-gestacional, ganho de peso materno, condições da assistência pré-natal e desfechos perinatais adversos entre puérperas adolescentes. Rev Bras Epidemiol 2012; 15(1):143-154.

13. Moraes ABM, Zanini RR, Riboldi J, Giugliani ERJ. Risk factors for low birth weight in Rio Grande do Sul State, Brazil: classical and multilevel analysis. Cad Saude Publica 2012; 28(12):2293-2305.

14. Guimarães AMDN, Betiol H, Souza L, Gurgel RQ, Almeida MLD, Ribeiro ERO, Goldani MZ, Barbiéri MA. Is adolescent pregnancy a risk factor for low birth weight? Rev Saude Publica 2013; 47(1):11-19.

15. World Health Organization (WHO). Reference 2007: Growth reference data for 5-19 years. Geneva. [internet] 2007. [acessado 2014 Maio 15]. Disponível em: http:// www.who.int/growthref/en/ 2007

16. Brasil. Ministério da Saúde (MS). Protocolos do Sistema de Vigilância Alimentar e Nutricional - SISVAN na assistência a saúde. Brasília: MS; 2008.

17. Institute of medicine (IOM). National Research Concil. Committee to Reexamine IOM Pregnancy Guidelines. Washington: National Academy Press; 2009.

18. Brasil. Ministério da Saúde (MS). Pré-natal e puerpério: atenção qualificada e humanizada. Manual Técnico. Brasília: MS; 2006. 
19. World Health Organization (WHO). International statistical classification of diseases and related health problems, tenth revision. Geneva: WHO; 1992.

20. Expert committee on the diagnosis and classification of diabetes mellitus. Report of the Expert Committee on the Diagnosis and Classification of Diabetes Mellitus. Diabetes Care 1997; 20(7):1183-1197.

21. International Association of Diabetes and Pregnancy Study Groups Consensus Panel. International association of diabetes and pregnancy study groups. Recommendations on the diagnosis and classification of hiperglycemia in pregnancy. Diabetes Care 2010; 33(3):676-682.

22. Santos MMAS, Barros DC, Nogueira JL, Baião MR, Saunders C. Impact of an intervention nutrition program during prenatal on the weight of newborns from teenage mothers. Nutr Hosp 2013; 28(6):1943-1950.

23. Padilha PC, Accioly E, Chagas C, Portela E, Silva CL, Sauders C. Birth weight variation according to maternal characteristics and gestational weight gain in Brazilian women. Nutr Hosp 2009; 24(2):207-212.

24. Rojas PFB, Francisco CC, Siqueira LFM, Carminatti APS. Fatores modificáveis associados ao baixo peso ao nascer da gravidez na adolescência. ACM Arq. Catarin. Med. 2012; 41(2):64-69.

25. Almeida AHV, Costa COM, Gama SGN, Amaral MTR, Vieira GO. Baixo peso ao nascer em adolescentes e adultas jovens na Região Nordeste do Brasil. Rev. Bras. Saude Matern. Infant. 2014; 14(3):279-286.

26. Lima MCBM, Oliveira GS, Lyra CO, Roncalli AG, Ferreira MAF. A desigualdade especial do baixo peso ao nascer no Brasil. Cien Saude Colet 2013; 18(8):24432452.

27. Ganchimeg T, Mori R, Ota E. Maternal and perinatal outcomes among nulliparous adolescents in low- and middle-income countries: a multi-country study. BJOG 2013; 120(13):1622-1630.

28. Organização das Nações Unidas (ONU). Declaração Mundial sobre a Sobrevivência, a Proteção e o Desenvolvimento da Criança. Nova Iorque: ONU; 1990.

29. Moreira TMM, Viana DS, Queiroz MVO, Bessa JMSB. Conflitos vivenciados pelas adolescentes com a descoberta da gravidez. Rev. Esc. Enferm. USP 2008; 42(2):312-320.

30. Phipps MG, Nunes AP. Assessing Pregnancy Intention and Associated Risks in Pregnant Adolescents. Matern Child Health J 2012; 16(9):1820-1827.

31. Shah SP, Balkhair T, Ohlsson A, Beyene J, Scott F, Frick C. Intention to Become Pregnant and Low Birth Weight and Preterm Birth: A Systematic Review. Matern Child Health J 2011; 15(2):205-216.

32. Dindaba Y, Fantahun M, Hindin M. The effect of pregnancy intention on use of the antenatal care services: systematic review and meta-analise. Reprod Health 2013; 10(50):1-9.

33. Neto MINP, Segre CSM. Análise comparativa das gestações e da frequência de prematuridade e baixo peso ao nascer entre filhos de mães adolescentes e adultas. Einstein 2012; 10(3):271-277.

34. Kingston D, Maureen H, Fell D, Chalmers B. Comparison of Adolescent, Young Adult, and Adult Women's Maternity Experiences and Practices. Pediatrics 2012; 29(5):e1228-e1237.
35. Viner RM, Ozer EM, Denny S, Marmot M, Resnick M, Fatusi A, Currie C. Adolescence and the social determinants of health. Lancet 2012; 379(9826):1641-1652.

36. Heilborn ML, Aquino EML, Bozon M, Knauth DR. O aprendizado da sexualidade: reprodução e trajetórias sociais de jovens brasileiros. Rio de Janeiro: Garamond, Fiocruz; 2006.

37. Viellas EF, Domingues RMSM, Dias MAB, Gama SGN, Theme Filha MM, Costa JV, Bastos MH, Leal MC. Assistência pré-natal no Brasil. Cad Saude Publica 2014; 30 (Supl. 1):S85-S100.

38. Meyer JD, Warren N, Reisine S. Racial and ethnic disparities in low birth weight delivery associated with maternal occupational characteristics. Am J Ind Med 2010; 53(2):153-162.

39. Vitolo MR, Bueno MSF, Gama CM. Impacto de um programa de orientação dietética sobre a velocidade de ganho de peso de gestantes atendidas em unidades de saúde. Rev Bras Ginecol Obstet 2011; 33(1):13-19.

40. Guerra AFFS, Heyde MEDV, Mulinar RA. Impacto do estado nutricional no peso ao nascer de recém-nascidos de gestantes adolescents. Rev Bras Ginecol Obstet 2007; 29(3):126-133.

41. Instituto Brasileiro de Geografia e Estatística (IBGE). Pesquisa de orçamentos familiares 2008-2009: análise do consumo alimentar pessoal no Brasil. Rio de Janeiro: IBGE; 2011.

42. Tielsch JM, Rahmathullah L, Katz J. Thulasiraj RD, Coles C, Sheeladevi S, Prakash K. Maternal Night Blindness during Pregnancy Is Associated with Low Birthweight, Morbidity, and Poor Growth in South India. J Nutr 2008; 138(4):787-792.

43. Leffelaar ER, Vrijkotte TGM, Van Eijsden M. Maternal early pregnancy vitamin $\mathrm{D}$ status in relation to fetal and neonatal growth: results of the multi-ethnic Amsterdam Born Children and their Development cohort. Br J Nutr 2010; 104(1):108-117.

44. Brasil. Ministério da saúde (MS). Guia alimentar para a população brasileira. 2a ed. Brasília: MS; 2014.

45. Barker DJP. Fetal origins of coronary heart disease. BMJ 1995; 311(6998):171-174.

46. Conde-Agudelo A, Belizán JM, Lammers C. Maternal-perinatal morbidity associated with adolescent pregnancy in Latin America. Cross-sectional study. Am J Obstet Gynecol 2009; 192(2):42-49.

Artigo apresentado em 20/01/2016

Aprovado em 16/08/2016

Versão final apresentada em 18/08/2016 